\title{
Towards Cooperative Sustainability
}

\author{
Wouter $\operatorname{Los}^{(\otimes)}(\mathbb{D}$ \\ Faculty of Science, University of Amsterdam, 1098XH Amsterdam, The Netherlands \\ w.los@uva.nl
}

\begin{abstract}
The inescapable question in the ENVRIplus project is how to sustain all achievements after the end of a collaborative project. Considering that each individual research infrastructure as cooperating in the ENVRIplus project has its own legal entity with dedicated governance and management, the challenge is to agree on modes of cooperation to keep tools and services of common interest up to date and operational. This chapter starts with the views of stakeholders, more specifically the views of scientific bodies, policy bodies, and of the infrastructure managers and operators who have to keep a lot of balls in the air. The sustainability plan has to consider the influences from external developments such as the European Strategy Forum on Research Infrastructures (ESFRI), and the emergence of a European Open Science Cloud (EOSC). The chapter discusses ENVRI strategic views, position, and future challenges.
\end{abstract}

Keywords: Sustainability $\cdot$ Governance $\cdot$ Planning

\section{Challenges}

As demonstrated in this book, many experts from many different research infrastructures worked together to develop novel tools supporting users of environmental research infrastructures in data production or to access, retrieve, and analyse existing data. All these data represent the dynamics of our environment with respect to the planetary crust, marine and freshwater bodies, the atmosphere, the impact of solar fluctuations, and the buffering role of the living environment/biodiversity. Apart from enhancing the services of each individual research infrastructure as they pertain to these environmental components, it is increasingly important to benefit from their combined services. The ambition of the cooperating research infrastructures is to provide scientists with the tools to work across traditional disciplinary boundaries and to discover, extract and analyse/model selected data as dispersed across many different sources and in many different formats. The cooperating research infrastructures tackled these ambitions by developing operational tools and services. Moreover, this provided the basis for bringing together the facilities, resources and services in support of the scientific community for innovative research and improved informed environmental policies. 


\subsection{Expectations of Scientific Bodies}

Developing the new services was strongly promoted by European funding under contracts for two successive projects ENVRI (2011-2014) [5] and ENVRIplus (2015-2019) [6], and was leveraged by national and other domestic funding. Such catalysing incentives proved to be crucial to foster cooperation between previously separated research infrastructure communities. Since the beginning of the 21st century, the European Strategy Forum on Research Infrastructures (ESFRI) brought together national policy and scientific stakeholders to consider and to promote the establishment of new research infrastructures in scientific areas requiring innovations for breakthroughs ${ }^{1}$. ESFRI showed itself to be very effective in promoting intergovernmental agreements for supporting the construction and operation of new research infrastructures at European and sometimes at the global scale in all scientific disciplines. Despite the challenging process to come to agreements within the scientific community and between supporting governments, a growing number of European research infrastructures managed to reach an agreement with and amongst funding countries, and started construction and successively entered their operational phases.

The ENVRI and ENVRIplus projects were conditional for sharing experiences, for sorting out solutions for common technical problems, and for providing guidance to support users requesting data and services from more than a single data source. In addition, the cooperation in these projects tackled common issues with respect to for example access services to multiple research infrastructures, ethical issues, training and exchange of staff, and common strategy development at the management level. These could not be developed in isolation, because attention had to be paid to the relationship with parallel developments in GEOSS ${ }^{2}$ and Copernicus, the European Union's environmental observation programme offering information services based on satellite observations and in situ data. Joint workshops assisted in defining each one's work area with at one side the research infrastructures as data generators and at other sides GEOSS and Copernicus as portals for access to the data as relevant for their successive missions. Still, a seamless connection in between them is not obvious as the requirements by the scientific users of research infrastructures, and the policy-oriented requirements of GEOSS and Copernicus users are not similar.

The League of European Research Infrastructures (LERU) published in 2017 a report on four golden principles for enhancing the quality, access and impact of research infrastructures [1]. First, a smart funding strategy is needed for research infrastructures to remain competitive, and to be leading and agile regarding further technological developments. Second, mechanisms should be developed to encourage cooperation, especially cross-border, thereby helping to avoid unnecessary duplication. Third, it is required to create a more robust and better-connected European network of research infrastructures. Fourth, the academic community should play a leading role in planning and operating the research infrastructures. Indeed, these four principles touch on important and also sensitive issues. Scientists expect that the research infrastructures are leading in new technical developments and related user services. However, such a risk-bearing

\footnotetext{
${ }^{1}$ https://www.esfri.eu.

2 http://www.geoportal.org.
} 
approach is very different from the more conservative risk-avoiding attitude of funding bodies (often ministries). This intrinsic tension complicates the sustainability of novel tools and services as described in this book, especially when this would require funding from different resources (countries, institutions and individual infrastructures). Most environmental research infrastructures are inherently distributed, dictated by the need to collect data and observations locally from around the planet. This may be an advantage for financing research infrastructures as it allows for domestic funding solutions rather than (only) funding a not-national European or international organisation. The disadvantage is however that it often prohibits the second principle above on avoiding unnecessary duplication through cross-border cooperation.

\subsection{Expectations of Scientific Bodies}

Several policy bodies addressed the challenges of sustaining research infrastructures, and of international cooperation. A strong example is the OECD Global Science Forum (GSF) with its mandate to address scientific issues that require global solutions ${ }^{3}$. Much attention has been and still is on research infrastructures, especially internationally funded infrastructures, and the international access to their facilities. The GSF promotes the principle that the world's best researchers should have access to the best infrastructure facilities, and that these facilities benefit from their innovative engagement. Continuous activity of the GSF is to identify policies and procedures that can strengthen the sustainability and the effectiveness of the functioning of research infrastructures during their entire lifecycle (including their dismantling or potential reuse). Priority targets are to lower initial investment and operating costs by improved standard planning and business models; to accelerate construction and implementation phases by better-adapted planning procedures; and to realise effective planning, budgeting and implementation of human-resources and controlled optimization of the running costs for RIs, considering that operational performance will lead to sustainable, attractive and productive research environments at both single-site and distributed RIs. However, this on-going activity did not give much attention to the implications of cooperation to promote the interoperability of research infrastructures.

In Europe, the initiative to implement a European Open Science Cloud (EOSC) puts emphasis on generic services for open access to data and related data interoperability ${ }^{4}$. This initiative is focusing on research outputs in general, and not specifically targeting research infrastructures. Nevertheless, the collaborative data services from research infrastructures are offering the strongest use cases in the EOSC development. The European Commission published in 2018 a "Call for Action Report" asking attention for critical issues to ensure the sustainability of research infrastructures [2]. The report, drafted as a Staff Working Document of the European Commission, recognises that sufficient funding is crucial for sustaining research infrastructures and their cooperation, but concludes that sustainability of RIs goes well beyond funding only, touching upon several dimensions such as scientific excellence, socio-economic impact and innovation. This is quite true for the environmental domain since societal and scientific challenges

\footnotetext{
3 http://www.oecd.org/sti/inno/global-science-forum.htm.

${ }^{4} \mathrm{https}: / /$ www.eosc-portal.eu.
} 
require full attention by the cooperating research infrastructures for tackling environmental complexity and its socio-economic impact of global change. Regrettably, the EC Staff Working Document only addresses the sustainability of individual research infrastructures and not their cooperative efforts which are beneficial from the viewpoints of interoperability, user support, and efficiency. Clearly, ENVRI enters unknown territory in the process to sustain its cooperative efforts, tools and services. ENVRI does not enter this unknown territory alone; however, since cluster-projects in other scientific domains meet similar challenges, for example, the CORBEL cooperative project of research infrastructures in the life sciences, which offers more integrated access to data resources required for biomedical research ${ }^{5}$.

\subsection{Keeping a Lot of Balls in the Air}

In the context explained above, sustaining the growing interoperability of research infrastructures is not an easy dedication for the management of collaborating individual infrastructures. One should consider that the emphasis in the last decade was on establishing new research infrastructures in Europe, and the understandable implication is that the infrastructure's management is primarily concerned with its own internal business. The EC financial support for fostering cooperation in between environmental research infrastructures was attractive to explore and develop common practices, tools, and services, but sometimes this was considered a distraction from the internal infrastructure's challenges. It takes time to appreciate efficiency benefits from cooperation or joint external marketing, as well to manage expectations in regard to keeping control of internal power versus advantages of delegating tasks to cooperative efforts. Currently, the infrastructure's view on organising data is changing. From putting data processing within a 'walled garden' (where many tools are brought together within a single curated platform), the new approach is a data 'marketplace' approach, where many tools are made available as micro-services within a wider market allowing users to find, compare, learn from others, and negotiate their preferred solutions or service level agreements. Apart from dealing with user demands, the management also has to deal with diverging interests of its national funders. Some of these funding bodies prefer to focus their policies on one or a few research infrastructures, while others don't want to bother with individual initiatives and are encouraging merging efforts to avoid fragmentation. The next paragraph reports about the process that the European environmental research infrastructures followed to sustain their cooperative efforts.

\section{The Making of a Sustainability Plan}

Environmental research infrastructures in Europe gained in the last decade more insight on how to work together and to benefit from cooperative efforts. A key principle was to foster engagement of all European-level research infrastructures, independent of any European or other funding. The management of such infrastructures engaged in regular

5 https://www.corbel-project.eu/home.html. 
meetings of a common advisory panel, the Board of Environmental Research Infrastructures (BEERi) ${ }^{6}$ [9]. This Board entered a process to agree on sustaining the results of the ENVRIplus project after the end of this project. One approach was to identify crucial common services, products and other results to be sustained after the end of the project, and by which organisations. This "bottom-up process" focused on the results relevant for all research infrastructures. A number of ENVRIplus partners are prepared to sustain these results with continued service provision. However, not all required tasks could be taken up by individual organisations. Covering these tasks brings into consideration a kind of collaborative organisational structure. This is a "top-down" process, focusing on the future ENVRI: the perspective for the infrastructures at the level of joint cooperation after the end of the funded ENVRIplus project.

The bottom-up process required the analysis of each ENVRIplus result in order to assess its relevance for all cooperating research infrastructures, and to negotiate with developers who have ownership and responsibility for sustaining them. In contrast, the top-down approach implied that infrastructure managers had to consider modes of future cooperation.

\subsection{The Bottom-Up Process to Identify Tools and Services of Common Interest}

The partners in the ENVRIplus project are best positioned to identify the common services, products and other results to be sustained after the end of the project. The focus here is primarily on work package (WP) results that are relevant for all research infrastructures. Results that are only relevant for one or a few Research Infrastructures will have to be dealt with by these infrastructures themselves. As a first step, the WP leaders were asked to consider which developed products/services should be sustained, and why. The results were categorised as follows:

- Intellectual: e.g. standards, concepts and reference docs.

- Networks: e.g. active communities, expert groups, BEERI and peer review mechanisms.

- (Online) Services/Distribution channels: e.g. interoperable data, web services, training and helpdesks.

- Physical infrastructure and software: e.g. computing environments, e-infrastructure and common virtual labs.

- Branding: Selected products/services requiring ENVRI branding as well as joint publicity and communication.

As a next step, the WP leaders were asked to clarify the ownership of results, applicable licenses, value propositions, required maintenance costs, and related annual costs. The identification of ownership of project results is important since the owners are in principle expected to take up the responsibility to sustain these results after the end of the project. Sustaining results does not only imply the maintenance and updates of services and products but also their accessibility and user support including training. The project's Grant Agreement states that (joint) owners may grant non-exclusive licenses to third

$\overline{6}$ https://www.envriplus.eu/beeri/. 
parties to exploit jointly-owned results. Each project partner had to seriously consider what would the wisest and most attractive way to proceed.

The process to collect the required information followed a number of steps as visualised in Fig. 1.

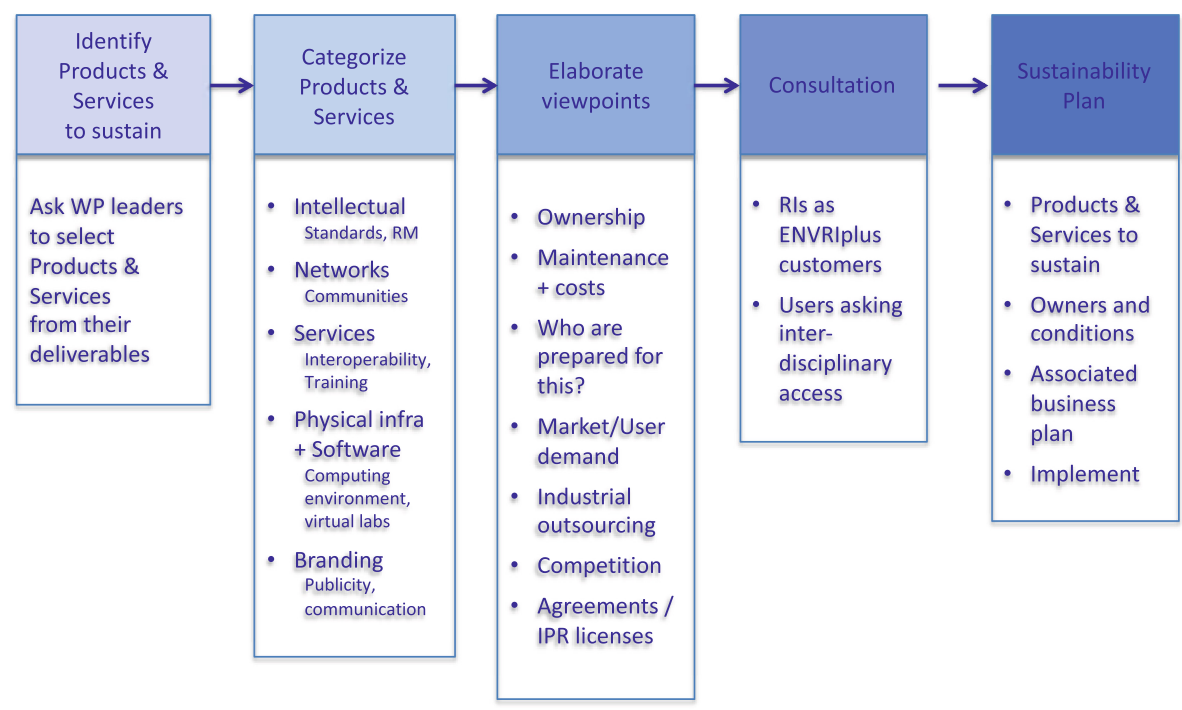

Fig. 1. Process steps to identify project results to be sustained.

Finally, this process ended up in an extensive overview of key results to be sustained at the ENVRI cluster level, and with the following details:

Who are the owners of products/services to sustain?

- Who are the owners (organisations) for each identified product or service?

- Are these owners ready to take organisational responsibility for sustaining the products or services?

- Alternatively, are agreements in place to transfer ownership (or license it) to a different organisation?

\section{Value proposition}

- Why is the product or service important/valuable for the research infrastructures?

- Which are the key user groups?

- How to advertise and provide support for deploying the product or service.

\section{Required maintenance and other activities in addition to annual costs}

- Which kinds of activities would be required to maintain, update, advertise, and provide user support and to provide training for the product or service? 
- What would approximately be the total annual costs for these activities?

This process was important to get all involved partners thinking about the future of their developed products. It was necessary to create awareness on any required agreements with the concerned partners to sustain their results for future use in the ENVRI community. But also on the question, if such agreements should be established with each separate research infrastructure, or with a joint ENVRI structure acting on behalf of the research infrastructures. The latter simplifies the picture and would allow for a common license model for ENVRIplus results. With this perspective, a joint indexed catalogue of all services and tools may become a reality. The catalogue should also provide contact details for getting the specific information and on associated training.

\subsection{The Top-Down Process to Conclude on a Joint ENVRI Service}

Different from the bottom-up analysis of the ENVRIplus results to be sustained, the topdown view considers how the research infrastructures want to proceed as a cooperative community. As for the question on the purpose and structure of a sustained ENVRI, the Implementation Roadmap for the European Open Science Cloud (EOSC) offers interesting considerations for establishing a strong ENVRI community [3]. This Roadmap identifies six main action lines to ensure the successful implementation of EOSC:

- Architecture: Create a pan-European federation of existing and future data infrastructures and resources. Bringing European research data infrastructures together will be a great improvement in their current state of fragmentation.

- Data: Foster the development of professional practices in research data management based on Findable, Accessible, Interoperable, and Reusable (FAIR) principles.

- Services: Make EOSC services available through a single access channel to all European researchers regardless of their discipline or location.

- Access and Interfaces: Simplify the use of data across different disciplines.

- Rules of participation: Set out the rights, obligations and accountability of EOSC stakeholders.

- Governance: Ensure EU leadership in data-driven science and adapt to new governance challenges.

The 2017 ESFRI report on the long-term sustainability of research infrastructures also presents arguments to foster a continued ENVRI community. Three recommendations are specifically relevant for the ENVRI cluster cooperation:

- Harmonise and integrate the operation of research infrastructures and einfrastructures.

- Demonstrate the economic and wider benefit to society of research infrastructures.

- Coordinate at National and European levels.

A top-down view may focus on promoting the impact of the ENVRI cooperation for multidisciplinary research, on addressing global change challenges, and on influencing policy and political decisions. It implies that prior to investigating any preferred 
organizational ENVRI structure, a common view should exist on the future purpose of the ENVRI community. Agreed priorities based on shared visions, values, goals and roles, will assist in sorting out preferred options for the future structure of ENVRI. Another top-down view is related to the efficiency benefits that the cooperating research infrastructures are expecting from a continued ENVRI cooperation. Which operational activities could be better organised within a common ENVRI community, rather than being addressed in each infrastructure separately?

\subsubsection{ENVRI Collaborative Strategy}

ENVRI, as a cooperation of European environmental research infrastructures, started in the first half of the 2010 decade with a common strategic view serving as guidance for organising joint project activities. The strategy was based on the vision to provide scientific support for a holistic understanding of our planet and its behaviour, processes, feedbacks, and fluxes. The challenge is then to contribute to developing an environmental system model, a framework of all interactions within the Earth System, from solid earth to near space. Many of the urgent challenges we are facing (such as climate change, energy use, water availability, food security, land degradation, hazards and risks, life in megacities, and human health) are closely related to complex interactions in the environment. Whilst each individual research infrastructure is concerned with its own domain of interest, it was thought imperative to find robust yet lightweight means to integrate various operations across research infrastructures to serve an increasingly multidisciplinary scientific community and to help to address the urgent societal challenges. To this end, three resources 'capitals' were identified as strategic targets within a conceptual model.

- Technological Capital: Capacity to measure, observe, compute, and store data, with technologies, software, and analytical and modelling capabilities.

- Cultural Capital: Open access to data, services in between RIs, requiring rules, licenses, citation agreements, IPR agreements, machine-machine interactions, workflows, metadata, data annotations, etc.

- Human Capital: The specialists to make it all work, with also generalists overseeing more than only their own discipline.

The ENVRIplus project focused on implementing these 'capitals', and this book reports the results in relation to common data issues. The next step is to benefit from the developed 'capitals' to address the above grand challenges. Discussions between the infrastructure managers revealed two main messages for an updated strategy. First, reconfirmation that ENVRI - as a large-scale cluster of European environmental research infrastructures -contributes to the societal challenges by providing high-quality multidisciplinary research data, services and expertise in a systemic way to mitigate societal risks. Second, that ENVRI aims to become a globally recognised cluster with strong international links and an attractive service portfolio for researchers, the private sector and policy-makers. This position has its implications for sustaining the common tools, services and other products as described in this book. As such, it assists in concluding about any future ENVRI organization. The next paragraph reports about the process followed to conclude on this. 


\subsubsection{The Process Towards a Joint Position on the ENVRI Organization}

Section 1.3 explained why it is for the management of research infrastructures, not an easy question to conclude about the role and organization of ENVRI in the next decade. While many research infrastructures are primarily concerned with their own construction and/or are consolidating their operations, it is understandable that they are less focused on common ENVRI interests. Nevertheless, they all appreciate the advantage of positioning some common activities at the ENVRI level. In addition, they feel that an ENVRI entity should take care of overseeing the agreements to sustain the results from the finished ENVRIplus project (the bottom-up process in Sect. 2.1).

In order to facilitate the discussion about alternative views, a consultation and interactive process was organised to promote consensus building on a common perspective. This process addressed the future of ENVRI as a cooperation of all recognised European research infrastructures. Subsets of research infrastructures may agree on other cooperation modes, but ENVRI is meant for the full infrastructure community. As such "mixed" models are always possible. The discussions focused on preferred approaches from 2025 on. Two consecutive anonymous surveys collected the preferences and opinions of each research infrastructure as associated with the ENVRIplus project. In between the two surveys, a moderated workshop discussed the pros and cons of alternative options to organise the future ENVRI, while taking into account the growing consensus of a common ENVRI strategy.

\subsubsection{Considered Organisational Options}

There are different options to consider for a future ENVRI structure: finishing the ENVRI cooperation, proceeding with a network, or federated cooperation, forming a modest or more extensive common legal entity, or even merging into a joint ENVRI research infrastructure. The collaborating research infrastructures were asked to express their preferences and views in two subsequent surveys, with in between a workshop to discuss the options. The first survey dealt with all options summarised below, and the second one focused on the most favoured ones.

\section{A) Finishing the ENVRI cooperation}

This option would imply that each research infrastructure goes separately, as shown in Fig. 2. Or, that some may conclude to proceed in a cooperative way, while others are leaving the ENVRI community. Of course, there may be irregular joint meetings, but bringing an end to the ENVRI cooperation will most likely reduce the voice and impact of the environmental cluster.

\section{B) ENVRI network, without required commitments}

An ENVRI network could be considered when enough research infrastructures would prefer to meet regularly and consider joint activities, either at the management level or at operational levels, as shown in Fig. 3. A simple network can be organised on the basis of a Memorandum of Understanding (MoU) with some agreements on how to run the network. The MoU may have provisions on for example a rotating chair and secretariat, on the subjects to be discussed or initiated, and on how each infrastructure may offer 


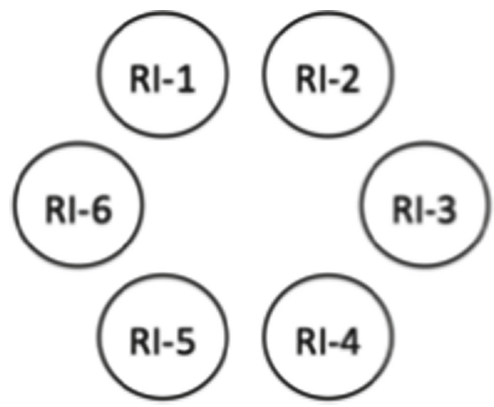

Fig. 2. No cooperation; each research infrastructure goes its own way.

in-kind contributions for the benefit for all. The latter may include the preparation of joint project proposals.

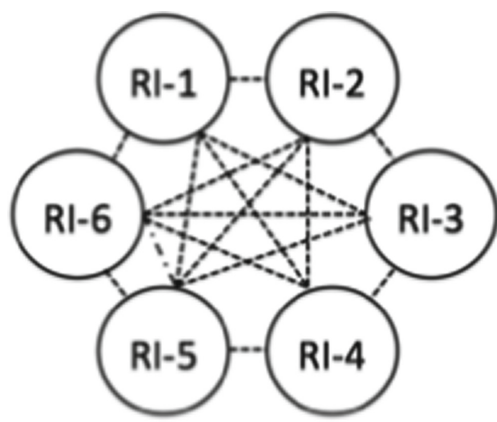

Fig. 3. Network cooperation without commitments.

\section{C) Federated structure with a joint consortium agreement}

Similar to collaborative projects, the research infrastructures agree to work together within a Consortium Agreement on specific topics. Figure 4 shows the basic idea. It will state in which areas will be cooperated, how this will be governed and managed, and how any financial contributions from partners for joint activities will be arranged. Depending on the agreement, one or more individual infrastructures may be assigned with the task of chair, secretary and treasurer.

\section{D) Establish an ENVRI legal body with limited roles}

This would be a legal body with limited tasks and powers, mainly for organising meetings and for organizing publicity, as shown in Fig. 5. It is a cheap option, and has the advantage of a united "place", both physically and on the Internet with a clear signal of the presence of ENVRI. Such a 'small' legal body should at least facilitate a secretariat and repository of joint undertakings. It may be considered to have in the legal body an independent director in charge, overseen by a board drawn from the cooperating research infrastructures. 


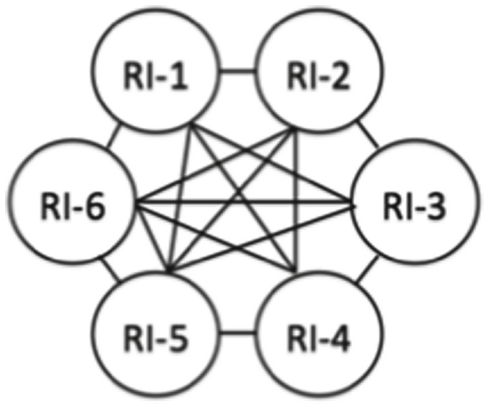

Fig. 4. No cooperation; each research infrastructure goes its own way.

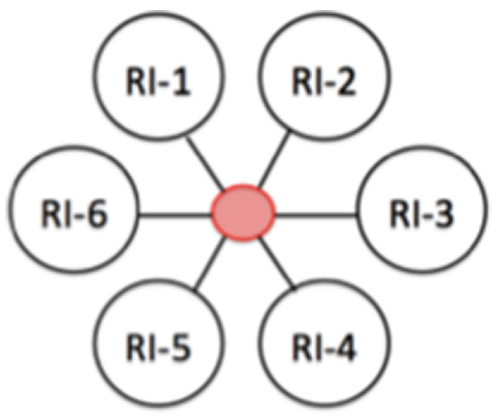

Fig. 5. Common legal body (in red) with limited roles.

E) An ENVRI legal body taking up common tasks for the research infrastructures

The common tasks are the ones that can more efficiently and/or less costly be operated from the legal entity. As a consequence, the legal body will employ its own staff, and costs are to be covered by fees paid by the involved RIs. The Board of the legal entity (with representatives of the cooperating RIs) decides on the tasks that can better be operated by the legal entity. Figure 6 shows the basic idea.

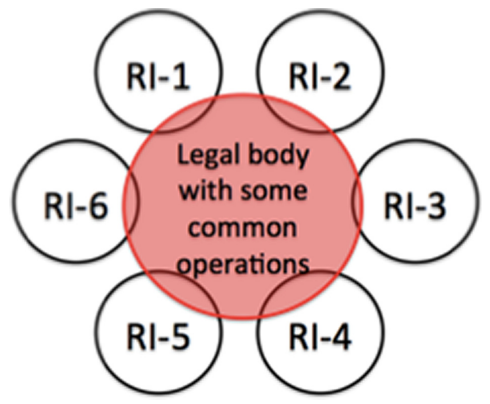

Fig. 6. A legal body (in red) taking up common tasks. 


\section{F) Merge the individual infrastructures in a joint research infrastructure}

This would a united legal ENVRI acting as an umbrella organisation of federated research infrastructures as working units, as shown in Fig. 7. Each individual infrastructure will continue its normal operations, while an overarching board takes care of the common interests. This board may appoint supervisory committees from the relevant communities for each RI. This is a similar set-up as for CERN and EMBL where different research facilities are operated within a common legal body.

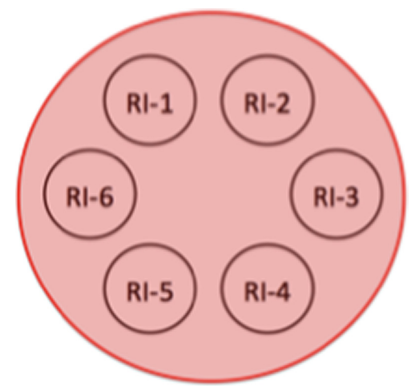

Fig. 7. A joint research infrastructure with merged facilities.

\section{Conclusions and Recommendations}

\subsection{Next Steps in the ENVRI Community}

The surveys and discussions about collaboration in a cluster of environmental research infrastructures revealed that nobody is in favour of finishing the ENVRI cooperation. In the long term, this would imply an agreement to establish an ENVRI cooperative body. The far-reaching option of merging all infrastructures within a single overarching research infrastructure was rejected. As for the intermediate considered options, the favoured approach is to plan for a legal body taking up a few common tasks, but to proceed through a step-by-step process: first, to collect signed Letters of Intent by each infrastructure, confirming the willingness to continue cooperation and the agreement to draft a Consortium Agreement (CA) matching with the above option c; next to agree on entering a joint consortium based on the $\mathrm{CA}$, and to experience how this would evolve. This has to underpin a future commitment to study and prepare a possible legal body with allocated tasks to be defined in detail.

Clearly, this perspective is important to promote the togetherness within the ENVRI community and to demonstrate the joint ENVRI dedication to external stakeholders. To this end an ENVRI business plan is in preparation, highlighting its focus, the benefits for collaborating in between research infrastructures and with external stakeholders, the commitments to agree upon, and the process towards further consolidation. In parallel, it is considered how to communicate with the political level about adapted and appropriate funding arrangements for the infrastructures in the ENVRI community. Considering the steps taken by the ENVRI community to reduce fragmentation in its infrastructure landscape, it makes sense to address the current fragmented arrangements for funding 
the European-scale research infrastructures. These are mainly funded through financial support by different combinations of individual countries. This picture is the result of elaborate negotiations with potentially interested countries, but with the complication that not any country will financially contribute to all environmental research infrastructures. This implies that huge transaction costs result from all these negotiations, and to be repeated every five years for another cycle of financial security. Another implication is that this fragmented funding structure makes it for the ENVRI community difficult to pursue a joint scientific and technical policy to address the grand societal and scientific questions.

\subsection{The ENVRI-FAIR Project}

The focus of the developing ENVRI business plan is primarily on securing the sustainability of jointly-developed tools and services, through securing service-levelagreements with the infrastructures or other organisations maintaining these tools and services, and by taking up some common tasks in the envisaged common ENVRI organisation. As for the tools and services mentioned in this book, the cooperating infrastructures are in a good position with the project ENVRI-FAIR, running for four years from the beginning of $2019^{7}$. This project is working on the uptake of the developed tools and services by individual research infrastructures and will facilitate the planned process towards a legal ENVRI entity. The business of the ENVRI-FAIR project is very much concerned with the parallel European developments for establishing a European Open Science Cloud (EOSC) [7]. The overarching goal of ENVRI-FAIR is to establish sustainable, transparent and auditable data services, for each step of the data lifecycle, compliant with the FAIR principles in the ENVRI community and connecting them to EOSC. Common policies, open standards, interoperability solutions, operational services, and stewardship of data on the basis of FAIR (Findable, Accessible, Interoperable, Reusable) principles require a common approach. The final goal is to prepare the open-access platform for interdisciplinary environmental research data in the European Research Area utilising EOSC. More specifically, the high-impact ambition of ENVRIFAIR is to establish the technical preconditions for the successful implementation of a virtual, federated machine-to-machine interface to access environmental data and services provided by the contributing research infrastructures. This ENVRI-hub is planned as a federated system of data policies and management, access platforms and virtual research environments. The system will be completely open-source, modular and scalable and build on the experience available in the consortium and already operational systems.

\subsection{Future Challenges}

The ENVRIplus project focused on bringing into place the capacities required for assisting researchers and other stakeholders in dealing with environmental challenges and providing key products for societal needs. Much progress has been made to improve cooperation in technology, and in a joint culture and in human capital. The next question is how to best benefit from the achievements as presented in this book. Clearly, the focus is than on supporting users in addressing the grand environmental challenges from solid earth to

\footnotetext{
${ }^{7}$ http://envri.eu/envri-fair/.
} 
near space. Many of the present urgent challenges are closely related to complex interactions in the environment. Whilst each research infrastructure is primarily concerned with its own domain of interest, another challenge is to integrate various operations across infrastructures, supporting the growing multidisciplinary scientific community. Indeed, the Earth system and problems related to the grand challenges are far too complex and interdependent to be studied from only a single perspective and supported by a single research infrastructure. New scientific developments require measurements covering the entire interlinked Earth system, and more integrated and interoperable infrastructure services enabling free access to and analysis of the gathered information. The latter is what ENVRIplus put in place, and was recognised by the ESFRI in its 2018 Roadmap [4], stated as follows (p. 14):

"The concept of multi-messenger research relies on exploiting diverse sources of information from different research methodologies to yield an integrated complementary ensemble of data that becomes the true insight on the phenomenon studied. Generalizing to all fields of research, we can recognise that a multi-messenger approach is already at work in domains like environmental sciences and life sciences, and that there is a high potential to address complex phenomena like grand societal and scientific challenges - e.g. climate change, population increase and differential ageing, food and energy sustainability - by using synergistically research infrastructures from all fields".

Possible approaches in this regard, and partly tackled by the new ENVRI-FAIR project, are the following:

- Challenge the scientific community to address the grand societal challenges with support by research infrastructures. An independent ENVRI scientific advisory body might be considered to define interdisciplinary research challenges, and to open up calls for inviting innovative researchers requiring advanced integrated services from the research infrastructures.

- Showcase the strengths and significance of ENVRI though user options to benefit from multiple sites and laboratory facilities, and of cross-use of experimental research platforms and vessels. An interesting consideration is to optimise the collaboration between industry, policy-makers and research infrastructures to promote a stronger impact of the research and innovation system, as also suggested in the ESFRI 2018 Roadmap.

- Be prepared to support interdisciplinary research, for example by providing data for a common minimal set of measurements/observations relevant for environmental variables regarding the Earth system, and a joint strategy to fill geographical gaps. An additional service could be to provide capacity for guiding and supporting interdisciplinary researchers requiring support from more than a single RI.

The benefit for the cooperating ENVRI research infrastructures is that they also will be challenged to provide joint services at the forefront of scientific discovery and societal impact. 
Acknowledgements. This work was supported by the European Union's Horizon 2020 research and innovation programme via the ENVRIplus project under grant agreement No 654182.

\section{References}

1. Väänänen, J., van Tienderen, P.: Four Golden Principles for Enhancing the Quality. Access and Impact of Research Infrastructures. LERU (2017). https://www.leru.org/publications/four-gol den-principles-for-enhancing-the-quality-access-and-impact-of-research-infrastructures

2. The European Commission: Sustainable European Research Infrastructures - a call for action. The European Commission (2017). https://ec.europa.eu/research/infrastructures/pdf/ ri_policy_swd-infrastructures_2017.pdf

3. The European Commission: Implementation roadmap for the European Open Science Cloud (EOSC). The European Commission (2018). http://ec.europa.eu/research/openscience/pdf/ swd_2018_83_f1_staff_working_paper_en.pdf

4. European Strategy Forum on Research Infrastructures: Strategy Report on Research Infrastructures (Roadmap 2018). ESFRI (2018). http://roadmap2018.esfri.eu/media/1066/esfri-roa dmap-2018.pdf

5. Chen, Y., Martin, P., Schentz, H., Magagna, B., Zhao, Z., Hardisty, A., Preece, A., Atkinson, M., Huber, R., and Legre. R.: A common reference model for environmental science research infrastructures. In: Proceedings of EnviroInfo 2013 (2013). http://enviroinfo.eu/sites/default/ files/pdfs/vol7995/0665.pdf

6. Zhao, Z., Martin, P., Grosso, P., Los, W., Laat, C. de, Jeffrey, K., Hardisty, A., Vermeulen, A., Castelli, D., Legre, Y., Kutsch, W.: Reference model guided system design and implementation for interoperable environmental research infrastructures. In: 2015 IEEE 11th International Conference on e-Science, pp. 551-556. IEEE, Munich (2015). https://doi.org/10.1109/eSc ience. 2015.41

7. Petzold, A., Asmi, A., Vermeulen, A., Pappalardo, G., Bailo, D., Schaap, D., Glaves, H.M., Bundke, U., Zhao, Z.: ENVRI-FAIR - interoperable environmental FAIR data and services for society, innovation and research. In: 2019 15th International Conference on eScience (eScience), pp. 277-280. IEEE, San Diego (2019). https://doi.org/10.1109/escience.2019. 00038, https://zenodo.org/record/3462816

Open Access This chapter is licensed under the terms of the Creative Commons Attribution 4.0 International License (http://creativecommons.org/licenses/by/4.0/), which permits use, sharing, adaptation, distribution and reproduction in any medium or format, as long as you give appropriate credit to the original author(s) and the source, provide a link to the Creative Commons license and indicate if changes were made.

The images or other third party material in this chapter are included in the chapter's Creative Commons license, unless indicated otherwise in a credit line to the material. If material is not included in the chapter's Creative Commons license and your intended use is not permitted by statutory regulation or exceeds the permitted use, you will need to obtain permission directly from the copyright holder.

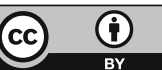

